\title{
JOINT SOURCE AND SENSOR LOCATION ESTIMATION
}

Norman L. Ows ley

Naval Underwater Systems Center New London, CT 06320

\section{ABSTRACT}

Expressions are presented for the minimum attainable bounds on the mean squared error for estimators of the range and bearing to a source and a parameter vector which specifies the receiving array sensor positions. It is assumed that noise corrupted measurements for estimating the sensor positions are available. For simple sensor array geometries, these results are compared to the corresponding performance when no source measurements are available. The results snow that having a source present at an unknown range and bearing can do little to improve the sensor position estimator performance. A polynomial model for the locus of the sensors in the receiving array is assumed.

\section{IN TRODUCTION}

Estimation of the range and bearing of a source relative to a receiving array of randomly perturbed sensors is a problem which has been previously considered by severa 1 authors [1-4]. In all of this previous work, the effect of the sensor perturbations on the source lacation parameters has been examined without considering the possibility that some type of measurement on the sensor positions might be available.

Moreover, the previous work used a statistical description of the sensor cartesian coordinates expressed in terms of the sensor coordinate mean values and cross-covariances. The question of joint source and sensor position estimation has not been previously considered.

We begin by assuming that a model for the locus of M discrete sensor locations exists in the form of a P-th order polynomial. Let this polynomial be

$$
y(x)=\sum_{p=1}^{p} a(p) x^{p}
$$

Consider Figure 1 which illustrates the location of the mth receiving array sensor at cartesian coordinates $\left(x_{m}, y\left(x_{m}\right)\right)$ and the source at polar coordinates $r$ and $\theta$. The output for the mth receiver is

$$
z_{m}\left(k_{1}\right)=s\left(k_{1}\right) e^{-j \omega \tau_{m}}+v_{m}\left(k_{1}\right)
$$

at frequency $\omega$, for $m=1,2, \ldots, M$ and $k_{1}=1,2, \ldots, k_{1}$. Both the signal and noise complex envelope samples $s\left(k_{1}\right)$ and $v_{m}\left(k_{1}\right)$ are taken from zero mean, independent and identically distributed complex gaussian random proceses with variances $S$ and $N$ respectively. The time delay $\tau_{m}$ referenced to zero at the coordinate system origin is given by $\tau_{m}=\left[r-r\left(x_{m}, y\left(x_{m}\right)\right)\right] / c$

$$
=\left[r-\sqrt{\left(r \cos \theta-x_{n}\right)^{2}+\left(r \sin \theta-y\left(x_{m}\right)\right)^{2}}\right] / c
$$

where $r\left(x_{m}, y\left(x_{m}\right)\right)$ is the distance from the source to the mth sensor. In Eq. (3), $c$ is the propagation velocity of the wavefront radiating cylindrically from the source.

Let the sensor locus polynomial coefficient vector a be defined as

$$
\underline{a}=[a(1), a(2), \ldots, a(P)]^{\top}
$$

Now we can write Eq. (1) in matrix form as

$$
y(x)=\underline{p}_{0}^{\top}(x) \underline{a}
$$

and its first derivative as

$$
\frac{\mathrm{dy}}{\underline{\mathrm{d}}(\mathrm{x})}=\underline{\mathrm{p}}_{1}^{\top}(\mathrm{x}) \underline{\mathrm{a}}
$$

where

$$
\underline{p}_{0}(x)=\left[x, x^{2}, x^{3}, \ldots, x^{P}\right]^{\top}
$$

and

$$
\underline{p}_{1}(x)=\left[1,2 x, 3 x^{2}, \ldots, P x^{P-1}\right]^{\top}
$$

Randomness in the receiving sensor locations is introduced by denoting the polynomial coefficient vector a as a random vector parameter. The number, $M$, of sensors receiving the signal is independent of the order of the locus polynomial, P. Next we assume that the noisy measurements

$z_{p q}\left(k_{2}\right)=\left.\frac{d y(x)}{d x}\right|_{x=x_{q}}+b(p)+v_{p q}\left(k_{2}\right)(N \geq q \geq p)$

of the sensor locus polynomial derivative are available. In Eq. (9), $k_{2}=1,2, \ldots, k_{2}$ is a time sample index, $b(p)$ is a random, zero mean gaussian measurement bias which is constant over 
the sequence of $K_{2}$ samples. The quantity $v_{p q}\left(k_{2}\right)$ is a zero mean statistically independent noise sample with variance $\sigma^{2}$. The random bias parameters are assumed to have the $N$-by- $N$ covariance matrix

$$
\begin{aligned}
\underset{\sim b}{R} & =E\left[\underline{b} \underline{b}^{\top}\right] \\
& =\sigma_{b}^{2} \underset{\sim}{I}
\end{aligned}
$$

where $b=[b(1), b(2), \ldots, b(N)]^{\top}, I_{N}$ is an $N-b y-N$ identity matrix and of is the mean squared value of $b(p)$ and is independent of $p$.

For complete generality the polynomial coefficient vector is assumed to be zero mean and gaussian with covariance matrix

$$
\begin{aligned}
\underset{\sim}{R} & =E\left[a \underline{a} a^{\top}\right] \\
& =\operatorname{diag}\left[\sigma^{2}(p)\right]
\end{aligned}
$$

Once we have established the joint parameter estimator performance using both sensor output Eq. (2) and sensor position Eq. (9) measurements, we want to obtain the performance of the estimator for a when only the position measurements of Eq. $(\bar{g})$ are used. Comparing these two performance bounds will determine the value of implementing a joint estimator as opposed to a two step process. The two steps would be to estimate a first using only the position measurements and, secondly, to estimate the source range and bearing using the sensor position locus as determined in the first step. The approach is to obtain the general performance measures for the two alternate estimators first. Then, specific two parameter problems are developed in detail to give insight into some fundamental relationships.

THEORY

We form a composite observation vector

$$
\underline{z}=\left[\begin{array}{c}
\underline{z}_{s}(1) \\
\underline{z}_{s}(2) \\
\vdots \\
\underline{z}_{s}\left(k_{1}\right) \\
--- \\
\underline{z}_{p}(1) \\
\underline{z}_{p}(2) \\
\vdots \\
\underline{z}_{p}\left(k_{2}\right)
\end{array}\right]=\left[\begin{array}{c}
\underline{z}_{s} \\
- \\
\underline{z}_{p}
\end{array}\right]
$$

of length $K_{1} M+K_{2}\left(N_{0}\right)$. The source

measurement vector $z_{s}\left(k_{1}\right)$ at time $k_{1}$ is the complex M-vector

$$
\underline{z}_{s}\left(k_{1}\right)=s\left(k_{1}\right) \underline{D}+\underline{v}_{s}\left(k_{1}\right)
$$

where the m-th element of the phase delay vector is $\exp \left(-j \omega \tau_{m}\right)$ and $\tau_{m}$ is specified by Eq.

(3). The sensor position measurement vector $\underline{z}_{p}\left(k_{2}\right)$ at time $k_{2}$ is the real (N!)-vector $\underline{z}_{p}\left(k_{2}\right)={\underset{\sim}{a}}_{a} \underline{a}+{\underset{\sim}{b}}_{b} \underline{b}+\underline{v}_{p}\left(k_{2}\right)$

where

$$
\stackrel{H}{a}_{a}=\left[\begin{array}{c}
P_{1}^{T}\left(x_{1}\right) \\
P_{1}^{\top}\left(x_{2}\right) \\
\vdots \\
P_{1}^{T}\left(x_{N}\right) \\
P_{1}^{T}\left(x_{2}\right) \\
P_{1}^{T}\left(x_{3}\right) \\
\vdots \\
P T \\
1 \\
\vdots \\
P T \\
1
\end{array}\right.
$$

and

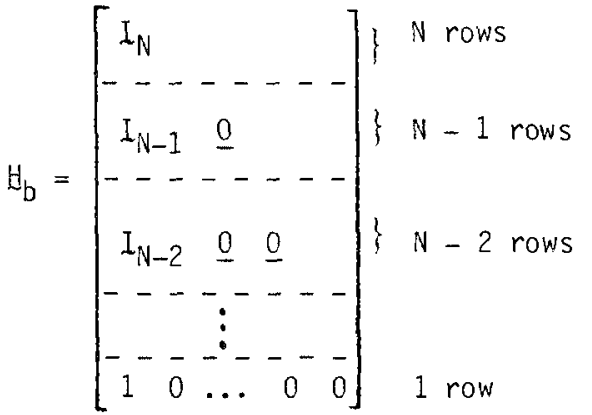

The position measurement noise vector, $v_{p}\left(k_{2}\right)$ is a sample vector of length $N$ ! from a statistically independent, zero mean and gaussian vector random process with covariance matrix

$$
\left.E\left[\underline{z}_{p}(k) \underline{z}_{p}(1)^{T}\right]=\sigma^{2}{\underset{\sim}{N}}_{N} \delta_{k}\right]
$$

The conditional probability density function for the composite measurement vector $\underline{z}$ and vector parameters $a$ and $b$ given the nonrandom range and beäring parameters is

$$
\begin{aligned}
& p(\underline{z}=\underline{\zeta}, \underline{a}=\underline{\alpha}, \underline{b}=\underline{\beta} / r, \theta)= \\
& p(\underline{z}=\underline{\zeta} / r, \theta, \underline{a}, \underline{b}) p(\underline{a}=\underline{a}) p(\underline{b}=\underline{B}) .
\end{aligned}
$$

We wish to make a joint estimate of the $(2+p+M)$ length parameter vector

$$
\begin{aligned}
& \underline{r}=\left[r_{1}, r_{2}, r_{3}, \ldots, r_{2+P+N}\right]^{\top} \\
& =\left[r, \theta, \alpha_{1}, \alpha_{2}, \ldots, \alpha_{p}, \beta_{1}, \beta_{2}, \ldots, \beta_{N}\right]^{\top} .
\end{aligned}
$$

The range, $r$, and bearing, $\theta$, are the free parameters about which no a priori knowledge is available. The remaining parameters have a known gaussian distribution. The Cramer-Rao theory [5] states that given the data vector $\underline{z}$, 
the best unbiased estimator $\hat{\underline{\gamma}}$ of the parameter vector $\gamma$ has the covariance matrix

$$
\operatorname{cov}[\hat{\gamma}]=\underline{J}(\underline{\gamma})^{-1} \text {. }
$$

The matrix $J(\underline{\gamma})$ is the Fisher information matrix whose elements are given by

$$
J_{i j}=-E\left[\frac{\partial^{2} \ln p(\underline{z}=\underline{\zeta}, \underline{a}=\underline{a}, \underline{b}=\underline{B} / r, \theta)}{\partial \gamma_{i} \partial \gamma_{j}}\right]
$$

where we can write

$p(\underline{z}=\underline{\zeta}, \underline{a}=\underline{\alpha}, \underline{b}=\underline{B} / r, \theta)=$

$p\left(\underline{z}_{S}=\underline{\zeta}_{S} / r, \theta, \underline{a}\right) p\left(\underline{z}_{p}=\underline{\zeta}_{p} / \underline{a}, \underline{b}\right) p(\underline{a}=\underline{\alpha}) p(\underline{b}=\underline{\beta})$.

The Fisher information matrix is given by the $2+p+M$ square matrix

$$
J(\underline{y})=J_{1}(\underline{\gamma})+J_{2}(\underline{\gamma})
$$

where

$$
J_{1}=\underbrace{\left.\left[\begin{array}{l:r}
A & 0 \\
0 & 0
\end{array}\right]\right\}}_{2+P} \begin{array}{ll}
2+P & M
\end{array}\}
$$

and

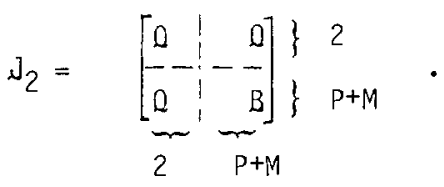

The $(2+P)-b y-(2+P)$ matrix $A$ has $i j-e l e m e n t ~ g i v e n$ by

$$
\begin{aligned}
& {[A]_{i j}=2 K_{1} \frac{\left(\frac{S}{N}\right)^{2} \omega^{2}}{1+M\left(\frac{S}{N}\right)}\left[M \sum_{m=1}^{M} \frac{\partial \tau_{m}}{\partial \gamma_{i}} \frac{\partial \tau_{m}}{\partial \gamma_{j}}-\right.} \\
& \left.\sum_{m=1}^{M} \frac{\partial \tau_{m}}{\partial \gamma_{j}} \sum_{m=1}^{M} \frac{\partial \tau}{\partial \gamma_{j}}\right] \text { for } 1 \leq i, \leq j \leq 2+P
\end{aligned}
$$

and the $(P+N)-b y-(P+N)$ matrix $B$ is given by

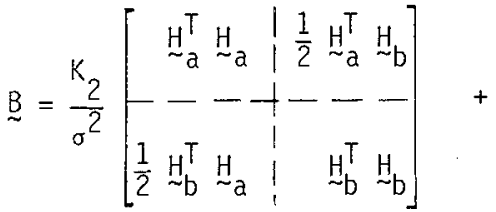

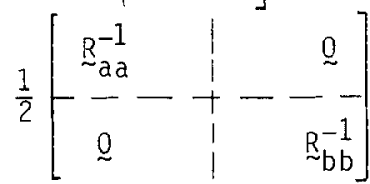

The Fisher matrix for estimation of the parameter vectors $a$ and $b$ with only the position measurements of $\mathrm{Eq}^{-}$. (9) is easily shown to be $\chi^{\prime}(\underline{a}, \underline{b})=B$ as defined by Eq. (27).

\section{EXAMPLES}

In the following examples, attention is focused on cases where $x_{m} \ll r, y\left(x_{m}\right) \ll r$ and $\theta \approx \pi / 2$. This corresponds to the situation where the source is not in the very near field and the source wavefront at the sensor array locus is represented we 1? by a quadratic in $x$. This is shown by approximating $r\left(x_{m}, y\left(x_{m}\right)\right)$ by a Taylor series truncated at the quadratic terms, while letting $\cos \theta=0$ to obtain

$$
\begin{aligned}
& \frac{\partial \tau_{m}}{\partial r_{1}}=\frac{\partial \tau_{m}}{\partial r}=x_{m}^{2} \sin \theta / 2 c r^{2} \\
& \frac{\partial \tau_{m}}{\partial \gamma_{2}}=\frac{\partial \tau_{m}}{\partial \theta}=-x_{m} \sin \theta / c \\
& \frac{\partial \tau_{m}}{\partial r_{2+p}}=\frac{\partial \tau_{m}}{\partial a(p)}=x_{m}^{p} \sin \theta / c
\end{aligned}
$$

The assumption $\theta \simeq \pi / 2$ effectively decouples the range and bearing estimation problems but still allows specific insight into the more general case. In the following, the receiving and position sensors are assumed to be at locations $x_{m}=d_{1}\left[m-\frac{M+1}{2}\right]$ and $x_{n}=d_{2}\left[n-\frac{N+1}{2}\right]$ respectively

Linear array: Let $y(x)=a(1) x$ with no bias in the position measurements, i.e., on $1 y r$ and $a(1)$ are to be estimated. Evaluation of the above gives

$$
\begin{aligned}
& \mathrm{J}_{11}=1 / \sigma_{r r}^{2}(\mathrm{~s}) \\
& \mathrm{J}_{12}=\mathrm{J}_{21}=0 \\
& \mathrm{~J}_{22}=1 / \sigma_{11}^{2}(\mathrm{~s})+1 / \sigma_{11}^{2}(\mathrm{p})
\end{aligned}
$$

where, with $d_{1}=L /(M-1), d_{2}=L /(N-1)$ and

$p=\left(\frac{S}{N}\right)^{2} /\left[1+M\left(\frac{S}{N}\right)\right]$,

we have

$$
\sigma_{r r}^{2}(s)=\frac{360 c^{2}}{K_{1} \rho}\left[\frac{r}{L}\right]^{4} \frac{(M-1)^{3}}{M^{2}(M+1)\left(M^{2}-4\right)} .
$$

This is the Cramer-Rao lower bound (CRLB) for the range estimator variance when the sensor positions are known exactly. Also

$$
\sigma_{11}^{2}(s)=\frac{6 c^{2}}{K_{1} \rho L^{2}} \frac{(M-1)}{M^{2}(M+1)}
$$

is the CRLB for the estimator of a(1) when no position measurements are used and

$$
\sigma_{11}^{2}(p)=\sigma^{2} / K_{2} N
$$

is the CRLB for the estimator of a(1) when no source measurements are used. The joint estimator of $r$ and $a(1)$ has the covariance matrix from Eq. (23) 


$$
\left[\begin{array}{cc}
\sigma_{r r}^{2} & \sigma_{r 1}^{2} \\
\sigma_{1 r}^{2} & \sigma_{11}^{2}
\end{array}\right]=\left[\begin{array}{ll}
J_{11} & J_{12} \\
J_{21} & J_{22}
\end{array}\right]^{-1}
$$

with the results

$$
\begin{aligned}
& \sigma_{r r}^{2}=\sigma_{r r}^{2}(s) \\
& \sigma_{r 1}^{2}=\sigma_{1 r}^{2}=0
\end{aligned}
$$

and

$$
\begin{aligned}
& \sigma_{11}^{2}=\sigma_{11}^{2}(p) /\left[1+\sigma_{11}^{2}(p) / \sigma_{11}^{2}(s)\right] \\
= & \sigma_{11}^{2}(p) /\left[1+\left(\sigma^{2} K_{1} \rho L^{2} M^{2}(M+1) / 6 K_{2} c^{2}(M-1) N\right)\right] .
\end{aligned}
$$

Thus, the range estimator is invariant to a(1) and the joint estimator provides no improvement in estimating the range. Moreover, the factor $\sigma^{2} \rho$ is typically so small that $\sigma_{11}^{2} \simeq \sigma_{11}^{2}(p)$ and

the source provides no significant information for estimating the sensor positions in terms of a(1). It would only be for extremely long observation time $K_{1}$ and high $(S / N)$ that the source could be exploited. Notice that the case $\left(1 / 0^{2}(1)\right)=0$ has been considered where in there is no prior knowledge of the coefficient a(1). Also, only the equality $q=p$ in $E q$. (9) is used.

Quadratic array: Let $y(x)=a(2) x^{2}$

with no bias in the position measurements, i.e. only $r$ and $a(2)$ are to be estimated. Now

$$
\begin{aligned}
& \mathrm{J}_{11}=1 / \sigma_{r r}^{2}(\mathrm{~s}) \\
& \mathrm{J}_{12}=\mathrm{J}_{21}=2 r^{2} / \sigma_{r r}^{2}(\mathrm{~s}) \\
& \mathrm{J}_{22}=4 r^{4} / \sigma_{r r}^{2}(\mathrm{~s})+\left(1 / \sigma_{22}^{2}(p)\right)
\end{aligned}
$$

where

$$
\sigma_{22}^{2}(p)=240 \sigma^{2} / K_{2} N(N+1)(N-1)\left(3 N^{2}-7\right)
$$

is the CRLB for the estimator of $a(2)$ given position measurements only. Evaluation of the joint estimator CRLB's as in Eq. (34) gives

$$
\sigma_{r r}^{2}=\sigma_{r r}^{2}(s)+4 r^{4} \sigma_{22}^{2}(p)
$$

and

$$
\sigma_{22}^{2}=\sigma_{22}^{2}(p) \text {. }
$$

Thus, the sensor position estimates are not improved at all by having the source present regard less of the ratio $(S / N)$. And, the source range estimator has the same performance $\mathrm{Eq}$. (40) with or without the joint estimator.

\section{DISCUSSION}

A general framework has been presented for evaluating the performance of a joint estimator of the location of a radiating source and the locations of sensors receiving the signal from the source. The Cramer-Rao theory allows for measurement of both received signal and noisy position data from the sensors. The specific cases of a sensor array with an unknown rotation and an unknown second order deformation without measurement bias have been considered and closed form results obtained. In neither case was there any significant value in jointly estimating the source and sensor location parameters unless there are long observation times and a high signal-to-noise ratio. It is felt that when deformation modes in the sensor array are predominantiy the same as those in the wavefront, i.e., linear and quadratic, then the joint estimator will provide little performance improvement over separate sensor and source position estimation procedures.

\section{REFERE NCES}

1. M. J. Hinich, "Bearing estimation using a perturbed linear array," J. Acoust. Soc. Am. 61, 1540-1549 (1977).

2. G. C. Carter, "Passive ranging errors due to receiving hydrophone position uncertainty," U.S. Navy J. Underwater Acoust. 29, 79-89 (1979).

3. P. M. Schultheiss and J. P. I anniello, "Optimum range and bearing estimation with randomly perturbed arrays," J. Acoust. Soc. Am. 68(1), July 1980.

4. P. M. Schulthe iss and E. Ashok, "Localization with arrays subject to sensor motion, " IEEE ICASSP 83, Boston, MASS, May 1983, Paper 8.12, pp. 371-374.

5. H. L. VanTrees, Detection and Estimation Theory, Wiley, New York, 1968, Part I, p. 84 .

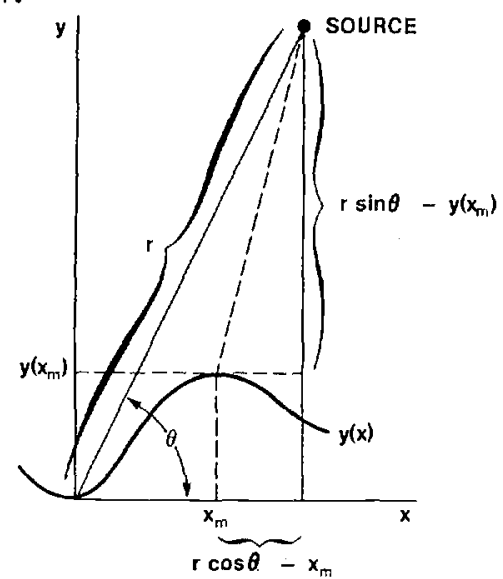

Figure 1. Sensor location $\left(x_{n}, y\left(x_{m}\right)\right)$ and source location $(r, \theta)$ geometry 